Published in final edited form as:

Pediatr Cardiol. 2017 March ; 38(3): 588-595. doi:10.1007/s00246-016-1552-y.

\title{
Timing and Mode of Delivery in Prenatally Diagnosed Congenital Heart Disease- an Analysis of Practices within the University of California Fetal Consortium (UCfC)
}

\author{
Shabnam Peyvandi $1{ }^{1,}$, Tina Ahn Thu Thi Nguyen $2,{ }^{*}$, Myriam Almeida-Jones $^{4}$, Nina Boe ${ }^{5}$, \\ Laila Rhee $^{5}$, Tracy Anton ${ }^{6}$, Mark Sklansky ${ }^{3}$, Maryam Tarsa ${ }^{6}$, Gary Satou $^{3}$, and Anita J. \\ Moon-Grady ${ }^{1}$ \\ ${ }^{1}$ Division of Pediatric Cardiology and the Fetal Treatment Center, University of California San \\ Francisco Benioff Children's Hospital, 550 16th street, 5th floor, San Francisco, CA, 94158 USA \\ ${ }^{2}$ Department of Obstetrics and Gynecology, David Geffen School of Medicine, University of \\ California, Los Angeles, CA, USA \\ ${ }^{3}$ Division of Cardiology, Mattel Children's Hospital and David Geffen School of Medicine, \\ University of California Los Angeles, Los Angeles, CA, USA \\ ${ }^{4}$ Division of Cardiology, University of California Irvine and Children's Hospital of Orange County, \\ Orange, CA, USA \\ ${ }^{5}$ Division of Maternal-Fetal Medicine, Department of Obstetric and Gynecology, University of \\ California, Davis, CA, USA \\ ${ }^{6}$ Division of Maternal Fetal Medicine, Department of Reproductive Medicine, University of \\ California, San Diego, CA, USA
}

\begin{abstract}
Prenatal diagnosis of critical congenital heart disease (CHD) is associated with decreased morbidity. It is also associated with lower birth weights and earlier gestational age at delivery. The University of California Fetal Consortium (UCfC) comprises five tertiary medical centers, and was created to define treatment practices. We utilized this consortium to assess delivery patterns and outcomes in subjects with prenatal and postnatal diagnosis of CHD. A retrospective cohort study was conducted on maternal-neonatal pairs diagnosed with complex CHD prenatally $(n=186)$ and postnatally $(n=110)$ from 2011 to 2013. Outcomes were assessed between groups after adjusting for disease severity. Prenatally diagnosed subjects were born earlier ( $38.1 \pm 0.11$ vs. $39 \pm 0.14$ weeks, $p=<0.001)$, and had lower birth weights (2853 \pm 49 vs. $3074 \pm 58 \mathrm{~g}, p=0.005)$ as
\end{abstract}

\footnotetext{
Shabnam Peyvandi: Phone 415-535-3277, Shabnam.peyvandi@ucsf.edu.

For The University of California Fetal Consortium (UCfC) Authors contributed equally

Compliance with Ethical Standards

Conflict of interest All authors declare no conflicts of interest.
}

Ethical Approval This article does not contain any studies with animals performed by any of the authors. All procedures performed in studies involving human participants were in accordance with the ethical standards of the institutional and/or national research committee and with the 1964 Helsinki declaration and its later amendments or comparable ethical standards. Informed consent was not required in this study due to the d-identified and retrospective nature of the data. A multi-institutional review board reliance registry provided approval for the study (IRB \#10-04093). 
compared to postnatal diagnosis. For every week increase in gestational age and $100 \mathrm{~g}$ increase in birth weight, length of stay decreased by $12.3 \pm 2.7 \%(p<0.001)$ and $3.9 \pm 0.9 \%(p<0.001)$. Subjects with prenatal diagnosis were more often born via cesarean both planned (35.6 vs. $26.2 \%$, $p=0.004)$ and after a trial of labor ( 13 vs. $7.8 \%, p=0.017$ ). Neonates with cesarean delivery trended toward a longer length of stay (2.6 days longer), and were born earlier as compared to other modalities ( $37.7 \pm 0.22$ weeks, $p=0.001)$. Management after prenatal diagnosis of CHD appears to have modifiable disadvantages for maternal and neonatal outcomes. The UCfC provides a platform to study best practices and standardization of care for future studies.

\section{Keywords}

Congenital heart disease; Prenatal diagnosis; Outcomes

\section{Introduction}

Prenatal detection of congenital heart disease (CHD) has increased during the last two decades with improvements in both ultrasonographic technology and increased rigor of screening in the obstetrical community [1, 2, 3, 4]. In particular, earlier detection of critical CHD requiring a neonatal intervention has allowed for planned deliveries at or near a tertiary hospital with a congenital cardiac surgery program and units equipped to manage these neonates [5, 6]. In addition, prenatal detection of critical CHD has been shown to improve the perioperative condition of these neonates $[7,8,9,10,11]$; however, multiple studies have failed to show a survival advantage to prenatal diagnosis [12 13]. Despite not leading to a survival advantage, prenatal diagnosis has been associated with better neurodevelopmental outcome in transposition of the great arteries (TGA) [14] as well as reduced risk of preoperative brain injury and improved trajectory of postnatal brain development in those with complex CHD [15].

This duality of prenatal diagnosis may be secondary to more complex disease being identified in utero; however, literature suggests that prenatal diagnosis is also associated with lower birth weights and earlier gestational age at birth $[8,16]$, two factors that have been linked to decreased survival, and poor neurologic outcomes [17, 18]. The etiology of lower birth weights and earlier delivery remains unknown, and may be influenced by modifiable factors such as practitioner education and comfort level with high-risk pregnancies involving fetal congenital heart disease.

The University of California Fetal Consortium (UCfC) comprises the five University of California campuses affiliated with university medical centers that offer prenatal diagnosis and treatment. The UCfC was established to better study pregnancies affected with maternal and fetal diseases, including congenital heart disease and to define treatment practices within our health system. We sought to harness this large statewide resource to describe delivery practices within the University of California medical centers performing prenatal diagnosis and postnatal management of CHD requiring neonatal intervention. Our aims were to assess delivery patterns, maternal outcomes, and neonatal outcomes in a well-characterized group 
of subjects with prenatal diagnosis of critical CHD and to compare them to a similar group of postnatally diagnosed subjects.

\section{Materials and Methods}

A retrospective cohort study was conducted on maternal-neonatal pairs with a prenatal or postnatal diagnosis of congenital heart disease born at or transferred to any of the five University of California Fetal Consortium (UCfC) sites from January 2011 to January 2013. A multi-institutional review board reliance registry provided approval for the study (IRB \#10-04093). All institutions participating in the UCfC are tertiary academic medical centers with perinatal, neonatal, and surgical services. Three centers perform complex neonatal cardiac surgical interventions, and two centers are affiliated with stand-alone children's hospitals that provide neonatal cardiac surgical services. Maternal-neonatal pairs with maternal, fetal, delivery, and neonatal outcomes data were included. Only those who required a neonatal operation within the first 30 days of life were included in this analysis. Pregnancies that were terminated and twin pregnancies were excluded.

Patients were identified via International Classification of Diseases, 9th revision codes, and site-specific research databases. Maternal and neonatal data collection was performed by chart review at each site. Primary outcomes included differences in mode of delivery, gestational age at delivery, birth weight, neonatal survival to 30 days, and length of hospital stay (LOS). Covariates included severity of CHD (using Risk Adjustment for Congenital Heart Surgery (RACHS) score [19]), need for intubation, and age at surgery. The RACHS score was assigned after retrospective review of each case including clinical and echocardiographic data by two senior cardiologists (A.M.G. and G.S.) blinded to outcome. Birth weight $z$ scores and percentiles were calculated based on the revised 2013 Fenton growth charts taking into account infant sex, gestational age at delivery, and birth weight [20]. Neonates with birth weights less than the 10th percentile for gestational age were considered small for gestational age (SGA).

\section{Statistical Analysis}

Standard descriptive statistics for continuous outcomes were used based on normality of data (Student $t$ test or Kruskal-Wallis). Proportions were compared with Fisher's exact test or the Chi-square test. Adjusted binary outcome values were computed using logistic regression. Adjusted actual delivery mode was computed using nominal logistic regression. Birth weight and $\log 10$ scale LOS were adjusted using linear regression since these outcomes had a normal distribution, and adjusted gestational age at delivery was computed using robust linear regression since gestational age at delivery did not follow a normal distribution. The Cox proportional hazard model was used to compute adjusted days to cardiac surgery. Statistical analysis was performed using SAS 9.4 (SAS Inc., Cary, NC) and R 3.2 (R project for statistical computing; https://www.r-project.org/).

\section{Results}

A total of 296 maternal-neonatal pairs were included in the study with 186 (63\%) neonates prenatally diagnosed and $110(37 \%)$ postnatally diagnosed. Demographic data are presented 
in Table 1. There was no difference between pre- and postnatal diagnosis groups in neonatal gender ( $p=0.39$ ) or in CHD severity (as graded by RACHS score, $p=0.18$ ). Maternal age in the prenatally diagnosed group was older as compared to the postnatally diagnosed subjects (30.8 and 27.9 years, respectively, $p=<0.001$ ). More prenatally diagnosed infants had extracardiac anomalies compared to those postnatally diagnosed (32.8 vs. $21.1 \%, p=$ 0.043 ), although a substantial number in each group had anomalies.

Prenatally diagnosed subjects were born at an earlier gestational age (38.1 weeks (IQR 3739 ) versus 39 weeks (IQR 38-39.6), $p=<0.001$ ) and had lower birth weights ( $2853 \pm 49$ vs. $3074 \pm 58 \mathrm{~g}, p=0.005$ ) as compared to their postnatal counterparts. Mean birth weight $z$ score was similar in both groups (prenatal: $-0.45 \pm 0.08$ vs. postnatal: $-0.33 \pm 0.08, p=$ 0.27 ), and both groups had a similar prevalence of SGA infants (prenatal: 22.4 vs. postnatal: $14.5 \%, p=0.06$ ). Patients with a postnatal diagnosis were more often intubated as compared to those prenatally diagnosed ( $p=0.037)$; however, there was no difference in initiation of PGE1 between the groups $(p=0.369)$. There was no difference between the groups in 30 day survival, survival to hospital discharge, or total length of hospital stay (Table 2).

Although all subjects eventually underwent a neonatal operation, postnatally diagnosed patients had surgery significantly sooner than prenatally diagnosed patients after adjusting for RACHS, gestational age at delivery, necessity for PGE1, and the presence of anomalies (Hazard ratio 1.40, $p=0.018$ ) (Fig. 1).

Given the significant differences between gestational age and birth weight between groups, we assessed whether these factors impacted total length of hospital stay. Consistent with prior reports we found an inverse relationship between gestational age at delivery and hospital LOS. After adjusting for RACHS, with every 1-week increase in gestational age, LOS decreased by $12.3 \pm 2.7 \%$ ( $p<0.001$ ) (Fig. 2). Similarly, after adjusting for RACHS, for every $100 \mathrm{~g}$ increase in birth weight, LOS decreased by $3.9 \pm 0.9 \%(p<0.001)$ (Fig. 3).

Subjects with a prenatal diagnosis were more often born via cesarean section, both planned (35.6 vs. $26.2 \%, p=0.004$ ) and after a trial of labor (either after a trial of labor including those with a previous cesarean delivery or as a primary cesarean without labor) (13 vs. 7.6\%, $p=0.017$ ) compared to postnatally diagnosed subjects (Table 3 ). To further assess practice patterns in mode of delivery, the planned vs. actual mode of delivery was analyzed in the prenatally diagnosed subjects (Table 4). Overall, the observed agreement rate was $56.4 \%$. Specifically, if the patient was scheduled for an induction of labor (IOL), 33\% ended up with a cesarean delivery (either without labor or after a failed trial of labor). Similarly, if the patient was intended for a normal spontaneous vaginal delivery (NSVD), 28\% ended up with a cesarean delivery (either without labor or after a failed trial of labor), thus contributing to the overall high rate of cesarean delivery in the prenatally diagnosed subjects (Table 4). Of the 23 subjects that had a change in delivery plan to a cesarean after a trial of labor, 14 had non-reassuring fetal heart tones, whereas in nine subjects, the reason was unclear. Similarly, of the 22 subjects that had a change in delivery plan to a cesarean without a trial of labor, eight had premature rupture of membranes, two had pre-eclampsia, and nine had nonreassuring fetal heart tones (the reason was unknown in three subjects). Interestingly, the presence of an extracardiac anomaly did not change delivery patterns. Among those with isolated CHD, planned cesarean delivery occurred in $38.7 \%$ and unplanned cesarean 
delivery occurred in $12.1 \%$. Similarly, in those with CHD with an extracardiac anomaly, $34.3 \%$ had a planned cesarean and $12.5 \%$ had an unplanned cesarean delivery.

Mode of delivery did not contribute to survival at discharge or 30 day survival in the prenatal ( $p=0.32$ and $p=0.11$ ) or postnatal diagnosis group ( $p=0.54$ and $p=0.86$ ). However, when assessing the entire cohort, there was a trend toward a longer hospital LOS for neonates born via cesarean delivery, suggesting that an effect of cesarean on LOS might have been demonstrable had the cohort been larger: hospital LOS was 6 days shorter for neonates born via NSVD or IOL (median 23.4 days, IQR 13-44.8) as compared to those born via cesarean delivery (median 29.4 days, IQR 16-65) ( $p=0.07$ ). This same trend existed after adjusting for RACHS, gestational age at delivery and birth weight. Hospital LOS for neonates born via NSVD or IOL (median 26.3 days, IQR 19.8-30.6) was 2.6 days shorter than those born via cesarean delivery (median 28.9 days, IQR 23.4-39.5) $(p=0.43)$. Finally, when assessing the distribution of gestational age at delivery by mode of delivery in the entire cohort, patients born via cesarean were born at a significantly younger gestational age ( $37.7 \pm 0.22$ weeks) as compared to those born via NSVD ( $39 \pm 0.11$ weeks) and IOL ( $38.8 \pm 0.31$ weeks), $p=$ 0.001 .

\section{Discussion}

Our results demonstrate significant differences between prenatal and postnatal diagnosis of severe CHD requiring a neonatal operation with respect to perinatal management and decisions around delivery planning. Although the severity of lesions was similar between the two groups, prenatally diagnosed subjects were born earlier with lower birth weights as compared to their postnatal counterparts. Furthermore, this is the first report to demonstrate differences in delivery practices in a large, well-characterized group of patients across multiple academic centers in the state of California, whereby prenatally diagnosed subjects were more often born via cesarean delivery.

Prior reports have suggested that prenatal diagnosis of CHD leads to early initiation of PGE1 and a "healthier" pre-operative state thus making prenatally diagnosed patients ideal candidates for a complex neonatal operation $[7,8,9,10]$. Interestingly, in our cohort, the prenatal diagnosis group waited longer to go to the operating room than the postnatal group, with postnatally diagnosed patients having their operation sooner. Despite not leading to a survival advantage $[12,13]$, the healthier pre-operative state probably leads to better longterm outcomes overall for those who survive. Specifically, studies have demonstrated improved neurodevelopmental outcomes and better brain health in subjects with TGA and HLHS that are diagnosed prenatally $[14,15]$. Conversely, prenatal diagnosis has also been associated with disadvantages in the form of earlier gestational age at delivery and lower birth weights [8]. Our findings mirror prior publications with prenatally diagnosed subjects being born "early-term" on average 1 week earlier than the postnatally diagnosed subjects. Although a difference of 1 week may not seem clinically significant, "early-term" birth in the CHD population has been associated with longer length of hospital stay, worse neurodevelopmental outcomes, and potentially increased mortality [17, 18]. In fact, our data demonstrate that for every 1 week increase in gestational age and for every $100 \mathrm{~g}$ increase in birth weight, total hospital length of stay decreases by 12.3 and $3.9 \%$, respectively. 
Optimizing pre-operative conditions may be critical to realizing the potential benefits of prenatal diagnosis; however, this appears in our cohort to be at the expense of optimal timing and mode of delivery.

In our cohort, $50 \%$ of mothers carrying a prenatally diagnosed fetus with CHD had a cesarean delivery, which is higher than the reported contemporary cesarean rate in California (30\%) (http://www.cdph.ca.gov), but also significantly higher than the rate of cesarean delivery in our postnatally diagnosed cohort. Although many of these were planned cesarean deliveries, even among mothers with a plan for IOL or spontaneous vaginal delivery after prenatal diagnosis, one-third resulted in a cesarean delivery. Our data and other studies demonstrate that mode of delivery does not impact neonatal outcomes in CHD [21, 22]. Thus, NSVD or IOL is safe and the preferred mode of delivery for these fetuses. However, despite this preference, a large percentage of mothers undergo cesarean delivery when carrying a fetus with CHD in the current era, even when an induction or NSVD was planned. Cesarean delivery is the modality of choice for certain maternal and fetal conditions [23, 24]; however, it is associated with increased maternal morbidities as compared to vaginal births in non-complicated pregnancies including abnormal placentation [25], higher rates of wound infection, and higher maternal rehospitalization rates [26]. Furthermore, these complications increase with repeated cesarean deliveries [25]. Our data are limited in that the precise clinical indication for planned cesarean delivery was not available. However, one would expect cesarean delivery rates in mothers carrying a fetus with CHD to mirror the general population, given the low prevalence of maternal co-morbidities in our cohort that would affect delivery modality. Although our data are largely based on practices at academic centers, large population-based studies have demonstrated no differences in cesarean delivery rates between academic and community hospitals [27].

We hypothesize that delivery planning in most of these cases is influenced by a desire to ensure the neonate is located at, or in close proximity to a center that can perform specific neonatal cardiac interventions. In the state of California, many patients travel long distances to obtain care in tertiary level medical centers such as our UC medical centers and affiliated hospitals. Thus, planning IOL at 39 weeks gestation allows for controlled-term delivery near a tertiary center. In our cohort, IOL was planned in $46 \%$ of prenatally diagnosed mothers. Although favorable for minimizing early-term birth and allowing for close proximity to tertiary centers, elective IOL has been associated with increased cesarean delivery rates [28, $29,30,31]$. In fact, our data demonstrate that 28 of the 85 pregnancies planned for IOL had a cesarean delivery (33\%). In addition, IOL prohibits the onset of natural labor and has been associated with increased length of stay $[28,29]$ and increased postpartum hemorrhage [32, 33] in comparison to NSVD. These factors all contribute to the estimated increase in cost and financial burden associated with IOL [34, 35]. In order to ensure safety and favorable outcomes for the neonate with a critical cardiac anomaly, it appears that maternal outcomes and cost are negatively affected. Not only does delivery mode impact maternal outcomes, our findings suggest that delivery mode may impact total length of hospital stay for the neonate requiring a cardiac operation with a trend toward a shorter hospital LOS for those born via NSVD or IOL. Although we did not collect actual maternal complications and length of stay in this study, an assumption that the mothers with cesarean deliveries stayed at least 1-2 days longer than the group with vaginal births can probably be made. We therefore 
speculate that investing in temporary relocation of some mothers to within close proximity of tertiary centers at least 1 week before the estimated date of delivery and allowing for spontaneous onset of labor rather than IOL or planned cesarean delivery may be more costeffective and beneficial to both the mother and fetus.

Our data have limitations in that we did not collect data on maternal LOS or complications. Although we adjusted for neonatal disease severity (RACHS), there may have been differences in CHD diagnosis severity or illness severity or subtle differences in the prenatally diagnosed patients that would not be corrected for using this methodology. In addition, it is plausible that the increased prevalence of extracardiac anomalies and the slightly higher prevalence of SGA in the prenatal diagnosis group influences our primary outcomes including GA at delivery and delivery mode. A larger sample size is needed to assess whether these factors play a significant role in maternal-neonatal outcomes in the context of CHD. Regarding planned cesarean deliveries, due to lack of access to the maternal prenatal records for patients delivering outside our systems, we were not able to collect whether cesarean deliveries were scheduled as elective repeat, done for malpresentation, or due to failed trial of labor after cesarean delivery. However, the relative incidence of these maternal indications should not have been different between the prenatally and postnatally diagnosed groups. Future studies can include prospectively assessing whether pregnancies with affected fetuses but without the above confounding factors are more likely to need C-section due to fetal intolerance of labor. This has been suggested by small retrospective studies evaluating the use of cardiotocography in labor showing a higher percentage of non-reassuring fetal heart rate tracings in CHD fetuses [36, 37]. In addition, lack of access to maternal prenatal records for those that delivered outside our systems did not allow us to collect data on the type and frequency of care they received. Our data reflect perinatal outcomes among patients diagnosed and treated at tertiary medical centers, thus may not be applicable to practices in a community-based model. The current analysis focuses on live born infants with CHD who underwent a neonatal operation. Thus, our study does not include an analysis of planned or actual delivery patterns among those with a fetal or perinatal demise. Finally, it is likely that socioeconomic status plays a role in both pregnancy and neonatal outcomes in this cohort, which was not collected for this analysis. Future studies assessing the impact of socioeconomic status on pregnancy and neonatal outcomes in the context of CHD will be performed.

\section{Conclusions}

In conclusion, management strategies after prenatal diagnosis of CHD, although beneficial in many areas, appear to have modifiable disadvantages. Education regarding the benefits of full-term delivery (for the neonate) and vaginal delivery after natural onset of labor (for the mother and neonate) may lead to better outcomes for maternal-fetal pairs and has the potential to decrease healthcare costs. Further studies will be needed to address whether temporary relocation of mothers close to tertiary centers, while awaiting the onset of labor will in fact leads to optimal outcomes as well as improve cost-effectiveness. Development of a multi-institutional clinical pathway and guidelines among the UCfC centers can assist in prospectively evaluating these remaining questions with a goal of standardizing care to improve outcomes and decrease cost. 


\section{Acknowledgments}

We would like to thank Jeffrey Gornbein, PhD (UCLA) for his assistance with statistical analysis and review of the manuscript.

Funding Dr. Peyvandi is supported by the NIH (P01 NS082330).

\section{References}

1. Fetal Echocardiography Task Force, American Institute of Ultrasound in Medicine Clinical Standards Committee, American College of Obstetricians and Gynecologists, Society for MaternalFetal Medicine. AIUM practice guideline for the performance of fetal echocardiography. J Ultrasound Med. 2011; 30:127-136. [PubMed: 21193716]

2. International Society of Ultrasound in Obstetrics and Gynecology. Carvalho JS, Allan LD, et al. ISUOG Practice Guidelines (updated): sonographic screening examination of the fetal heart. Ultrasound Obstet Gynecol. 2013; 41:348-359. DOI: 10.1002/uog.12403 [PubMed: 23460196]

3. Tometzki AJ, Suda K, Kohl T, et al. Accuracy of prenatal echocardiographic diagnosis and prognosis of fetuses with conotruncal anomalies. JAC. 1999; 33:1696-1701.

4. Allan LD, Sharland GK, Milburn A, et al. Prospective diagnosis of 1006 consecutive cases of congenital heart disease in the fetus. JAC. 1994; 23:1452-1458.

5. Donofrio MT, Moon-Grady AJ, Hornberger LK, et al. Diagnosis and treatment of fetal cardiac disease: a scientific statement from the American Heart Association. Circulation. 2014; 129:21832242. DOI: 10.1161/01.cir.0000437597.44550.5d [PubMed: 24763516]

6. Morris SA, Ethen MK, Penny DJ, et al. Prenatal diagnosis, birth location, surgical center, and neonatal mortality in infants with hypoplastic left heart syndrome. Circulation. 2014; 129:285-292. DOI: 10.1161/CIRCULATIONAHA.113.003711 [PubMed: 24135071]

7. Tworetzky W, McElhinney DB, Reddy VM, et al. Improved surgical outcome after fetal diagnosis of hypoplastic left heart syndrome. Circulation. 2001; 103:1269-1273. [PubMed: 11238272]

8. Kipps AK, Feuille C, Azakie A, et al. Prenatal diagnosis of hypoplastic left heart syndrome in current era. Am J Cardiol. 2011; 108:421-427. DOI: 10.1016/j.amjcard.2011.03.065 [PubMed: 21624547]

9. Mahle WT, Clancy RR, McGaurn SP, et al. Impact of prenatal diagnosis on survival and early neurologic morbidity in neonates with the hypoplastic left heart syndrome. Pediatrics. 2001; 107:1277-1282. [PubMed: 11389243]

10. Kumar RK, Newburger JW, Gauvreau K, et al. Comparison of outcome when hypoplastic left heart syndrome and transposition of the great arteries are diagnosed prenatally versus when diagnosis of these two conditions is made only postnatally. AJC. 1999; 83:1649-1653.

11. Friedberg MK, Silverman NH, Moon-Grady AJ, et al. Prenatal detection of congenital heart disease. J Pediatr. 2009; 155(26-31-31):e1.doi: 10.1016/j.jpeds.2009.01.050

12. Oster ME, Kim CH, Kusano AS, et al. A population-based study of the association of prenatal diagnosis with survival rate for infants with congenital heart defects. Am J Cardiol. 2014; 113:1036-1040. DOI: 10.1016/j.amjcard.2013.11.066 [PubMed: 24472597]

13. Wright LK, Ehrlich A, Stauffer N, et al. Relation of prenatal diagnosis with one-year survival rate for infants with congenital heart disease. Am J Cardiol. 2014; 113:1041-1044. DOI: 10.1016/ j.amjcard.2013.11.065 [PubMed: 24440326]

14. Calderon J, Angeard N, Moutier S, et al. Impact of prenatal diagnosis on neurocognitive outcomes in children with transposition of the great arteries. J Pediatr. 2012; 161(94-8):e1.doi: 10.1016/ j.jpeds.2011.12.036

15. Peyvandi S, De Santiago V, Chakkarapani E, et al. Association of prenatal diagnosis of critical congenital heart disease with postnatal brain development and the risk of brain injury. JAMA Pediatr. 2016; doi: 10.1001/jamapediatrics.2015.4450

16. Levey A, Glickstein JS, Kleinman CS, et al. The impact of prenatal diagnosis of complex congenital heart disease on neonatal outcomes. Pediatr Cardiol. 2010; 31:587-597. DOI: 10.1007/ s00246-010-9648-2 [PubMed: 20165844] 
17. Costello JM, Pasquali SK, Jacobs JP, et al. Gestational age at birth and outcomes after neonatal cardiac surgery: an analysis of the society of thoracic surgeons congenital heart surgery database. Circulation. 2014; 129:2511-2517. DOI: 10.1161/CIRCULATIONAHA.113.005864 [PubMed: 24795388]

18. Goff DA, Luan X, Gerdes M, et al. Younger gestational age is associated with worse neurodevelopmental outcomes after cardiac surgery in infancy. J Thorac Cardiovasc Surg. 2012; 143:535-542. DOI: 10.1016/j.jtcvs.2011.11.029 [PubMed: 22340027]

19. Jenkins KJ, Gauvreau K, Newburger JW, et al. Consensus-based method for risk adjustment for surgery for congenital heart disease. J Thorac Cardiovasc Surg. 2002; 123:110-118. [PubMed: 11782764]

20. Fenton TR, Kim JH. A systematic review and meta-analysis to revise the Fenton growth chart for preterm infants. BMC Pediatr. 2013; 13:59.doi: 10.1186/1471-2431-13-59 [PubMed: 23601190]

21. Peterson AL, Quartermain MD, Ades A, et al. Impact of mode of delivery on markers of perinatal hemodynamics in infants with hypoplastic left heart syndrome. J Pediatr. 2011; 159:64-69. DOI: 10.1016/j.jpeds.2011.01.004 [PubMed: 21414630]

22. Trento LU, Pruetz JD, Chang RK, et al. Prenatal diagnosis of congenital heart disease: impact of mode of delivery on neonatal outcome. Prenat Diagn. 2012; 32:1250-1255. DOI: 10.1002/pd.3991 [PubMed: 23080120]

23. Hannah ME, Hannah WJ, Hewson SA, et al. Planned caesarean section versus planned vaginal birth for breech presentation at term: a randomised multicentre trial. Term breech trial collaborative group. Lancet. 2000; 356:1375-1383. [PubMed: 11052579]

24. Minkoff H, Chervenak FA. Elective primary cesarean delivery. N Engl J Med. 2003; 348:946-950. DOI: 10.1056/NEJMsb022734 [PubMed: 12621140]

25. Silver RM, Landon MB, Rouse DJ, et al. Maternal morbidity associated with multiple repeat cesarean deliveries. Obstet Gynecol. 2006; 107:1226-1232. DOI: 10.1097/01.AOG. 0000219750.79480.84 [PubMed: 16738145]

26. Lydon-Rochelle M, Holt VL, Martin DP, Easterling TR. Association between method of delivery and maternal rehospitalization. JAMA. 2000; 283:2411-2416. [PubMed: 10815084]

27. Snyder CC, Wolfe KB, Loftin RW, et al. The influence of hospital type on induction of labor and mode of delivery. Am J Obstet Gynecol. 2011; 205(346):e1-e4. DOI: 10.1016/j.ajog.2011.05.004

28. Seyb ST, Berka RJ, Socol ML, Dooley SL. Risk of cesarean delivery with elective induction of labor at term in nulliparous women. Obstet Gynecol. 1999; 94:600-607. [PubMed: 10511367]

29. Maslow AS, Sweeny AL. Elective induction of labor as a risk factor for cesarean delivery among low-risk women at term. Obstet Gynecol. 2000; 95:917-922. [PubMed: 10831992]

30. Cammu H, Martens G, Ruyssinck G, Amy J-J. Outcome after elective labor induction in nulliparous women: a matched cohort study. Am J Obstet Gynecol. 2002; 186:240-244. [PubMed: 11854642]

31. Yeast JD, Jones A, Poskin M. Induction of labor and the relationship to cesarean delivery: a review of 7001 consecutive inductions. Am J Obstet Gynecol. 1999; 180:628-633. [PubMed: 10076139]

32. Sheiner E, Sarid L, Levy A, et al. Obstetric risk factors and outcome of pregnancies complicated with early postpartum hemorrhage: a population-based study. J Matern Fetal Neonatal Med. 2005; 18:149-154. DOI: 10.1080/14767050500170088 [PubMed: 16272036]

33. Vrouenraets FPJM, Roumen FJME, Dehing CJG, et al. Bishop score and risk of cesarean delivery after induction of labor in nulliparous women. Obstet Gynecol. 2005; 105:690-697. DOI: 10.1097/01.AOG.0000152338.76759.38 [PubMed: 15802392]

34. Kaufman KE, Bailit JL, Grobman W. Elective induction: an analysis of economic and health consequences. Am J Obstet Gynecol. 2002; 187:858-863. [PubMed: 12388964]

35. Allen VM, O'Connell CM, Farrell SA, Baskett TF. Economic implications of method of delivery. Am J Obstet Gynecol. 2005; 193:192-197. DOI: 10.1016/j.ajog.2004.10.635 [PubMed: 16021078]

36. Ueda K, Ikeda $\mathrm{T}$, Iwanaga $\mathrm{N}$, et al. Intrapartum fetal heart rate monitoring in cases of congenital heart disease. Am J Obstet Gynecol. 2009; 201(64):e1-e6. DOI: 10.1016/j.ajog.2009.03.015

37. Morikawa M, Endo D, Yamada T, et al. Electronic fetal heart rate monitoring in five fetuses with Ebstein's anomaly. J Obstet Gynaecol Res. 2014; 40:424-428. DOI: 10.1111/jog.12190 [PubMed: 24118438] 


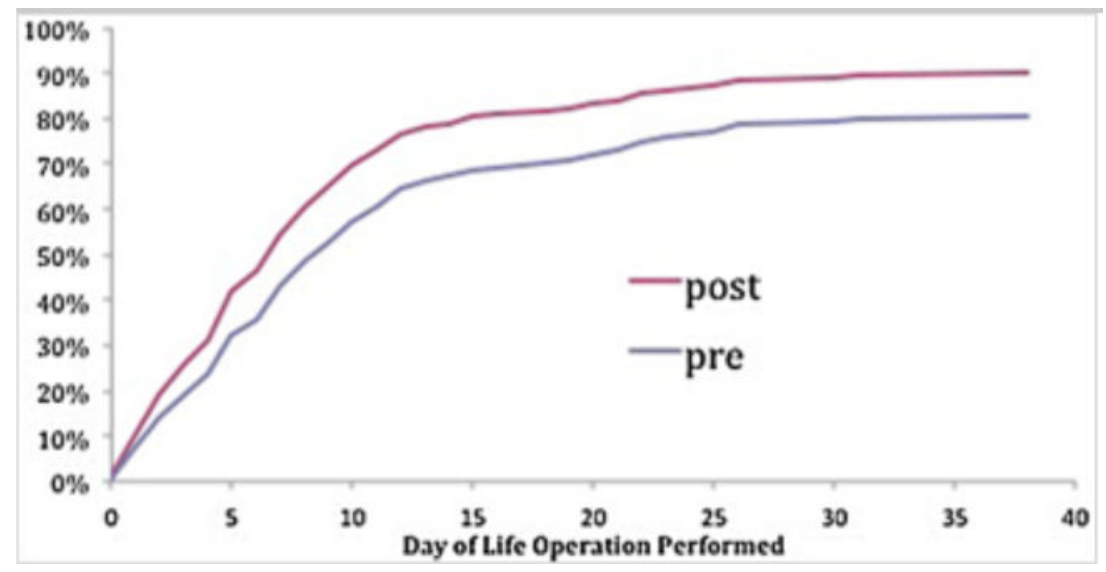

Fig. 1.

Time to surgery for prenatal vs. postnatal diagnosis of critical congenital heart disease.

Legend incidence curves for number of days to surgery in prenatal and postnatally diagnosed subjects. The $\mathrm{x}$-axis represents day of life when surgery was performed and the $\mathrm{y}$-axis represents percentage of neonates. After adjustment for RACHS, gestational age at delivery, necessity for prostaglandins and presence of non-cardiac anomalies, postnatally diagnosed subjects had their operation 1.4 times faster than those prenatally diagnosed (Hazard Ratio $=$ 1.4) 


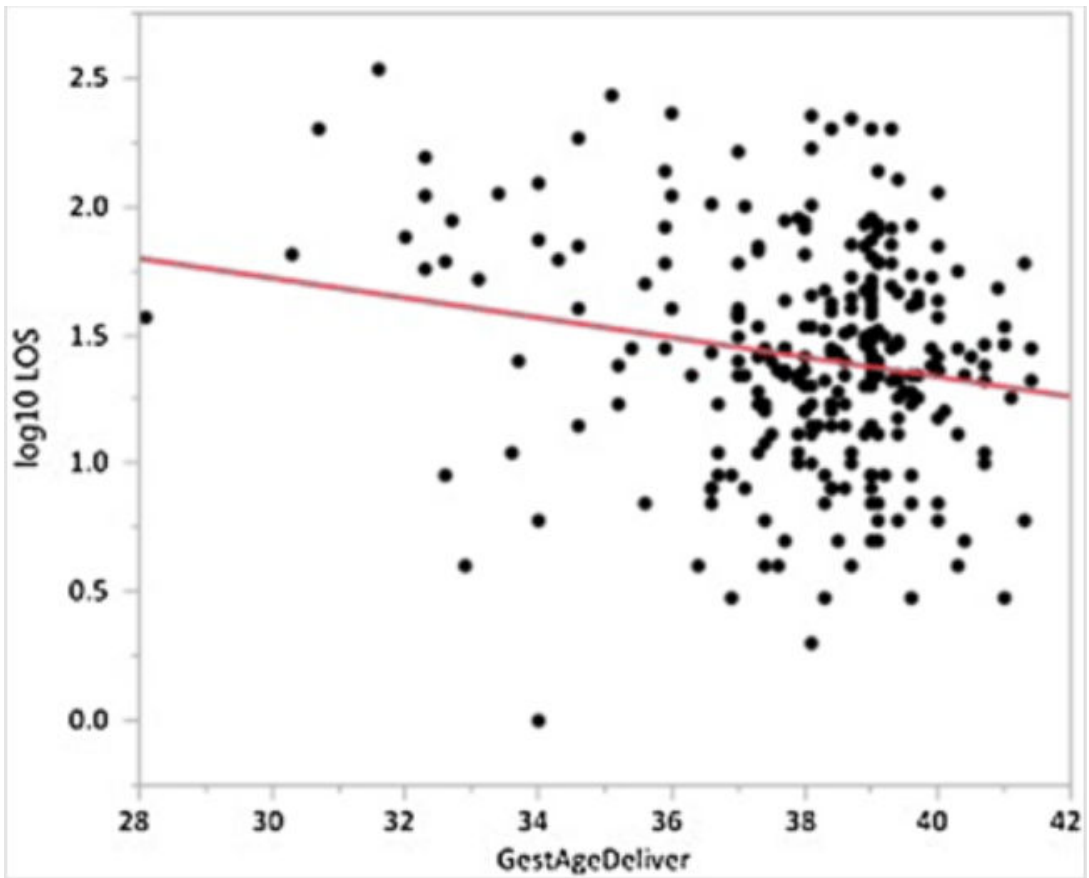

Fig. 2.

The correlation between gestational age at delivery and neonatal hospital length of stay. Legend the $\mathrm{x}$-axis represents gestational age at delivery in weeks and the y-axis represents the total hospital length of stay ( $\log _{10}$ transformed). After adjusting for RACHS (Risk Adjustment for Congenital Heart Surgery), with every 1-week increase in gestational age, length of stay decreased by $12.3 \%(p<0.001)$ 


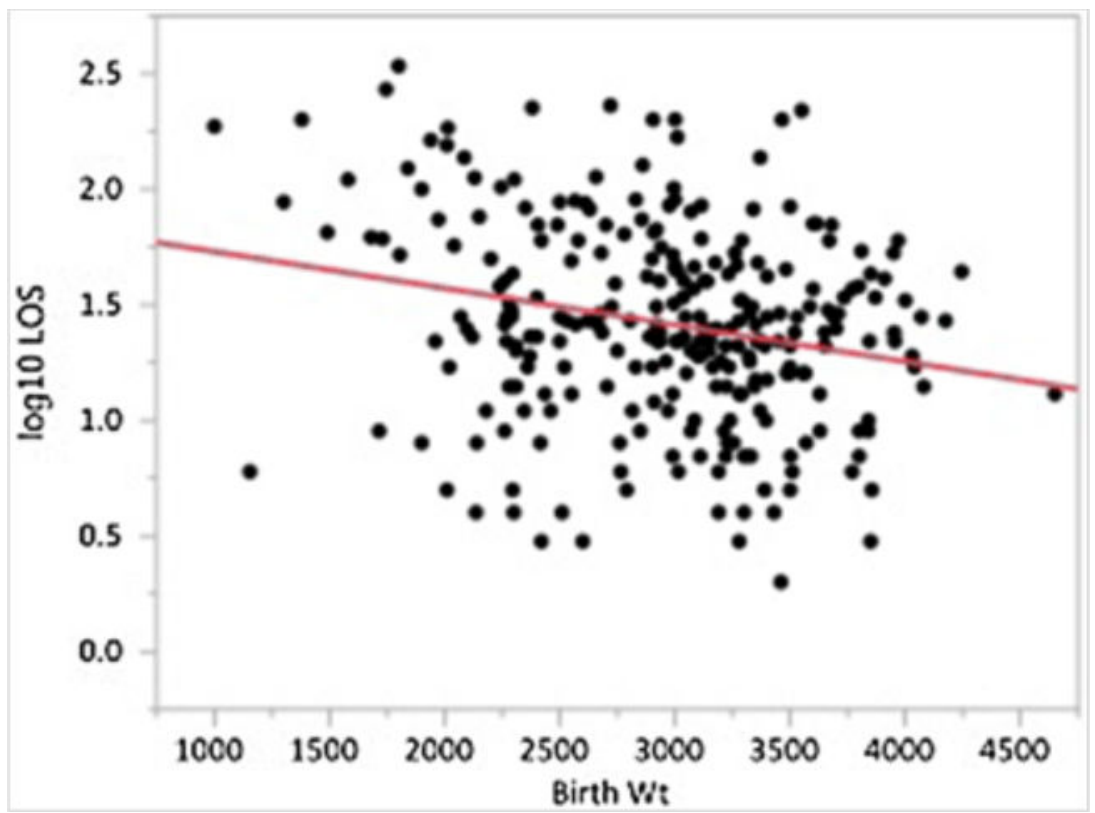

Fig. 3.

The correlation between birth weight and neonatal hospital length of stay. Legend the x-axis represents birth weight in grams and the $y$-axis represents total hospital length of stay $\left(\log _{10}\right.$ transformed). After adjusting for Risk adjustment for congenital heart surgery (RACHS), with every $100 \mathrm{~g}$ increase in birth weight, length of stay decreased by $3.9 \%(p<0.001)$ 


\section{Table 1}

Demographic data and baseline characteristics of study cohort

\begin{tabular}{|l|l|l|l|}
\hline & Prenatal Dx $(\boldsymbol{n}=\mathbf{1 8 6})$ & Postnatal Dx $(\boldsymbol{n}=\mathbf{1 1 0})$ & $\boldsymbol{p}$ value \\
\hline Maternal age $($ mean \pm SE) & $30.8 \pm 0.48$ & $27.9 \pm 0.60$ & $<0.001$ \\
\hline Male gender $n(\%)$ & $100(54.3 \%)$ & $66(60.0 \%)$ & 0.395 \\
\hline RACHS (median,IQR) & $3.0(2.0-4.0)$ & $3.0(2.0-4.0)$ & 0.178 \\
\hline Other anomalies $n(\%)$ & $61(32.8 \%)$ & $23(21.1 \%)$ & 0.043 \\
\hline
\end{tabular}

$R A C H S$ risk adjustment for congenital heart surgery score, $D x$ diagnosis, $S E$ standard error, $I Q R$ interquartile range 
Table 2

Neonatal outcomes in prenatal and postnatal diagnosis of CHD

\begin{tabular}{|l|l|l|l|}
\hline & Prenatal Dx $(\boldsymbol{n}=\mathbf{1 8 6})$ & Postnatal Dx $(\boldsymbol{n}=\mathbf{1 1 0})$ & $\boldsymbol{p}$ value \\
\hline EGA (median, IQR) & $38.1(37-39)$ & $39.0(38-39.6)$ & $<0.001$ \\
\hline & & $3074 \pm 58$ & 0.005 \\
\hline BW $z$ score (mean \pm SE) & $-0.45 \pm 0.08$ & $-0.33 \pm 0.08$ & 0.27 \\
\hline SGA $n(\%)$ & $42(22.4 \%)$ & $16(14.5 \%)$ & 0.06 \\
\hline Intubation $n(\%)$ & $66(35.7 \%)$ & $53(48.2 \%)$ & 0.037 \\
\hline PGE1 started $(n) \%$ & $119(65.8 \%)^{a}$ & $78(70.9 \%)$ & 0.369 \\
\hline Survival to discharge n $(\%)$ & $154(82.7 \%)$ & $97(88.2 \%)$ & 0.237 \\
\hline 30 d survival $n(\%)$ & $158(84.9 \%)$ & $102(92.6 \%)$ & 0.079 \\
\hline LOS (median, IQR) & $27.0(14-60)$ & $25.0(14-45)$ & 0.455 \\
\hline
\end{tabular}

$D x$ diagnosis, $E G A$ estimated gestational age, $B W$ birthweight, $L O S$ length of stay, $P G E 1$ prostaglandin E1, $S E$ standard error, $I Q R$ interquartile range, $S G A$ small for gestational age (defined as birth weight percentage less than the 10th percentile for gestational age)

a Data missing on five subjects 
Table 3

Maternal delivery mode in prenatal and postnatal diagnosis

\begin{tabular}{|l|l|l|l|}
\hline & Prenatal Dx $\boldsymbol{n}(\boldsymbol{\%})$ & Postnatal Dx $\boldsymbol{n}(\boldsymbol{\%})$ & $\boldsymbol{p}$ value \\
\hline NSVD & $65(35.5 \%)$ & $69(65.2 \%)$ & $<0.001$ \\
\hline IOL & $27(14.8 \%)$ & $3(2.8 \%)$ & $<0.001$ \\
\hline Cesarean after trial of labor/induction & $23(12.6 \%)$ & $8(7.6 \%)$ & 0.017 \\
\hline Cesarean planned & $68(37.2 \%)$ & $26(24.5 \%)$ & 0.004 \\
\hline Total & $183(100 \%)^{a}$ & $106(100 \%)^{a}$ & \\
\hline
\end{tabular}

$D x$ diagnosis, $N S V D$ normal spontaneous vaginal delivery, $I O L$ induction of labor

${ }^{a}$ Data missing for three subjects in prenatal diagnosis group and in four subjects for the postnatal diagnosis group 

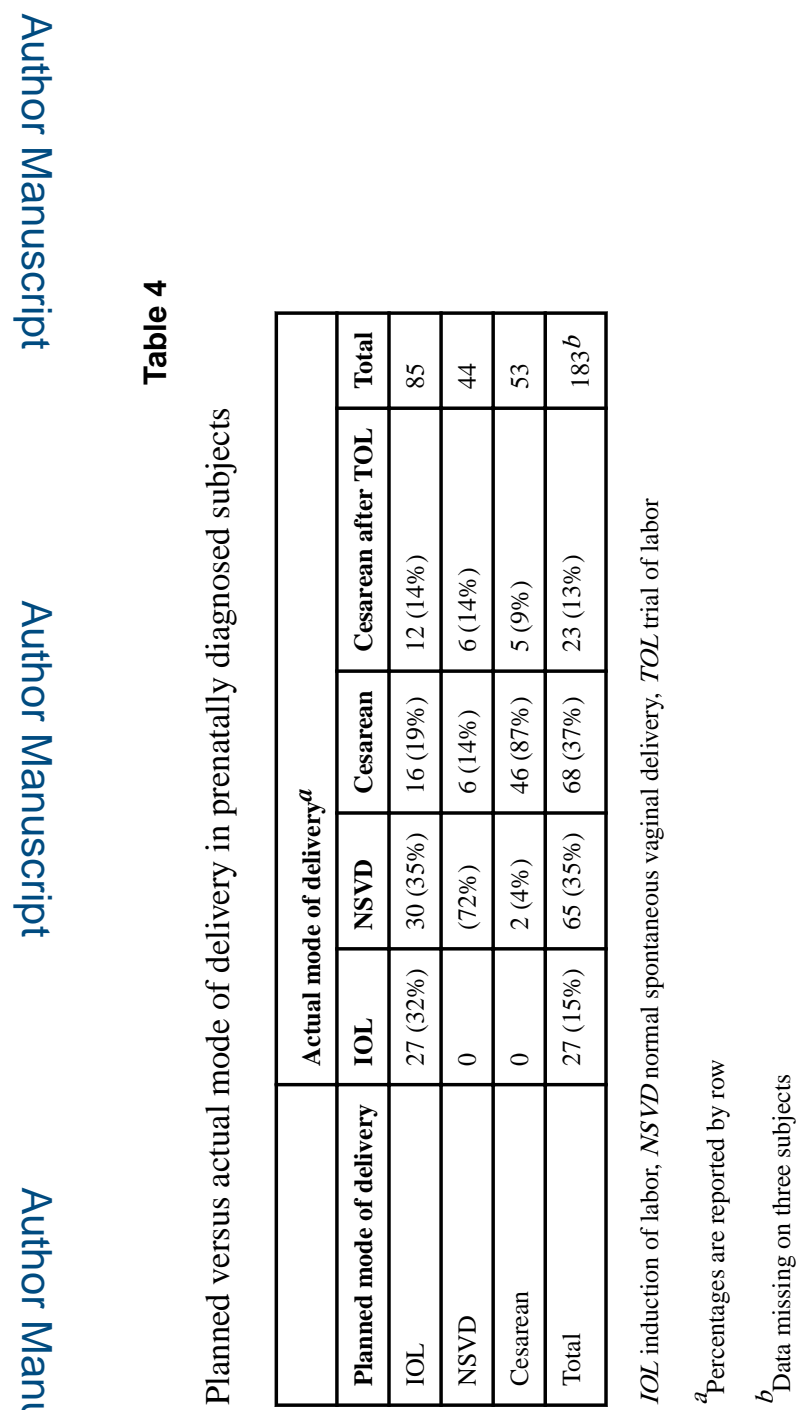

Pediatr Cardiol. Author manuscript; available in PMC 2018 May 25 